INLO-PUB-03/02

FAU-TP3-02-18

hep-th/0205116

\title{
Constituents of Doubly Periodic Instantons
}

\author{
C. Ford ${ }^{a}$ and J. M. Pawlowski ${ }^{b}$ \\ ${ }^{a}$ Instituut-Lorentz for Theoretical Physics \\ Niels Bohrweg 2, 2300 RA Leiden, The Netherlands \\ ford@lorentz.leidenuniv.nl \\ ${ }^{b}$ Institut für Theoretische Physik III, Universität Erlangen \\ Staudtstraße 7, D-91058 Erlangen, Germany \\ jmp@theorie3.physik.uni-erlangen.de
}

\begin{abstract}
Using the Nahm transform we investigate doubly periodic charge one $S U(2)$ instantons with radial symmetry. Two special points where the Nahm zero modes have softer singularities are identified as constituent locations. To support this picture, the action density is computed analytically and numerically within a two dimensional slice containing the two constituents. For particular values of the parameters the torus can be cut in half yielding two copies of a twisted charge $\frac{1}{2}$ instanton. Such objects comprise a single constituent.
\end{abstract}

Topologically non-trivial objects play a pivotal rôle in most confinement scenarios. A prominent example being the dual Meissner effect via the condensation of magnetic monopoles. To date, there is no gauge independent way of identifying the monopole content of a given gauge potential. However, some progress has been made with regard to instantons. In particular, charge one $S U(N)$ calorons, or periodic instantons, can be viewed as bound states of $N$ monopole constituents [1, 2]. This identification does not hinge on a particular gauge choice as the constituents are clearly visible as peaks in the action density. Presumably, similar results hold for 'higher' tori, that is, instantons on $\mathbb{T}^{2} \times \mathbb{R}^{2}$ (doubly periodic instantons), $\mathbb{T}^{3} \times \mathbb{R}$ and the four torus $\mathbb{T}^{4}$. In the latter case twists must play a rôle since untwisted charge one instantons do not exist [3].

There is an almost complete lack of explicit results for multiply periodic instantons in the literature. However, the existence of higher charge instantons on $\mathbb{T}^{4}$ was established by 
Taubes. More recently, doubly periodic charge one instantons have been discussed [4, 5]. In [5] doubly periodic charge one $S U(2)$ instantons with radial symmetry in the noncompact $\mathbb{R}^{2}$ directions were considered. Under the Nahm transformation these instantons are mapped to abelian potentials on the dual torus $\tilde{\mathbb{T}}^{2}$. Our basic approach is to start with these rather simple abelian potentials and then Nahm transform to recover the original $S U(2)$ instanton. This involves solving certain Weyl-Dirac equations; for each $x$ in $\mathbb{T}^{2} \times \mathbb{R}^{2}$ one has a different Weyl equation on $\tilde{\mathbb{T}}^{2}$. The Weyl zero modes were determined explicitly for a two dimensional subspace [5] of $\mathbb{T}^{2} \times \mathbb{R}^{2}$ (this subspace corresponds to the origin of $\mathbb{R}^{2}$ ), see also [6].

Although this falls short of a complete solution, this subspace is, by virtue of the radial symmetry, exactly where any constituents are expected to lie. In this letter we use the explicit zero modes to develop a constituent picture of the associated doubly periodic instantons. There are two points in the subspace where the zero modes have different singularity profiles. These are obvious candidates for constituent locations. The behaviour of the field strengths in the vicinity of the proposed constituent locations is investigated analytically and numerically. It is possible to arrange that the two constituents are identical lumps. If we further fix their separation to be a half period, the torus can be cut in two. In each half of the torus we have a twisted instanton of topological charge $\frac{1}{2}$ comprising a single constituent.

Before we specialise to $\mathbb{T}^{2} \times \mathbb{R}^{2}$ let us briefly recall how the Nahm transform is formulated on $\mathbb{T}^{4}$ [3]. Start with a self-dual anti-hermitian $S U(N)$ potential, $A_{\mu}(x)$, on a euclidean four-torus with topological charge $k$. A gauge field on $\mathbb{T}^{4}$ is understood to be an $\mathbb{R}^{4}$ potential which is periodic (modulo gauge transformations) with respect to $x_{\mu} \rightarrow x_{\mu}+L_{\mu} \quad(\mu=$ $0,1,2,3)$, the $L_{\mu}$ being the four periods of the torus. The next, apparently trivial, step is to turn the $S U(N)$ instanton into a $U(N)$ instanton by adding a constant $U(1)$ potential, $A_{\mu}(x) \rightarrow A_{\mu}(x)-i z_{\mu}$, where the $z_{\mu}$ are real numbers. We can regard the $z_{\mu}$ as coordinates of the dual torus, $\tilde{\mathbb{T}}^{4}$, since the shifts $z_{\mu} \rightarrow z_{\mu}+2 \pi / L_{\mu}$ can be effected via periodic $U(1)$ gauge transformations.

Now consider the $U(N)$ Weyl operator

$$
D_{z}(A)=\sigma_{\mu} D_{z}^{\mu}(A), \quad D_{z}^{\mu}(A)=\partial^{\mu}+A^{\mu}(x)-i z^{\mu}
$$

$\sigma_{\mu}=\left(1, i \tau_{1}, i \tau_{2}, i \tau_{3}\right)$ where the $\tau_{i}$ are Pauli matrices. Provided certain mathematical tech- 
nicalities are met $D_{z}^{\dagger}(A)=-\sigma_{\mu}^{\dagger} D_{z}^{\mu}(A)$ has $k$ square-integrable zero modes $\psi^{i}(x ; z)$ with $i=1,2, \ldots, k$. The Nahm potential is defined as

$$
\hat{A}_{\mu}^{i j}(z)=\int_{T^{4}} d^{4} x \psi^{i^{\dagger}}(x ; z) \frac{\partial}{\partial z^{\mu}} \psi^{j}(x ; z) .
$$

Here the zero modes are taken to be orthonormal. Remarkably, $\hat{A}(z)$ is a $U(k)$ instanton on the dual torus with topological charge $N$. If we execute a second Nahm transformation on $\hat{A}$, the original $S U(N)$ instanton will be recovered.

Formally, one can obtain the $\mathbb{T}^{2} \times \mathbb{R}^{2}$ Nahm transform by taking two of the periods, say $L_{0}$ and $L_{3}$, to be infinite. Given an $S U(N)$ instanton periodic with respect to $x_{1} \rightarrow x_{1}+L_{1}$, $x_{2} \rightarrow x_{2}+L_{2}$ its Nahm transform is

$$
\begin{array}{lll}
\hat{A}_{\mu}^{i j}(z)=\int_{T^{2} \times R^{2}} d^{4} x \psi^{i \dagger}(x ; z) \frac{\partial}{\partial z^{\mu}} \psi^{j}(x ; z) & \mu=1,2 \\
\hat{A}_{\mu}^{i j}(z)=\int_{T^{2} \times R^{2}} d^{4} x \psi^{i \dagger}(x ; z) i x_{\mu} \psi^{j}(x ; z) & \mu=0,3
\end{array}
$$

where the $\psi^{i}(x ; z)$ with $i=1, \ldots, k$ are orthonormal zero modes of $D_{z}^{\dagger}(A)$. Note that we may gauge $z_{0}$ and $z_{3}$ to zero. The Nahm potential is a self-dual $U(k)$ gauge field with $N$ singularities in $\tilde{\mathbb{T}}^{2}$ (periods $2 \pi / L_{1}$ and $2 \pi / L_{2}$ ).

One can regard $\hat{A}_{1}(z)$ and $\hat{A}_{2}(z)$ as the components of a two dimensional gauge potential, and combine $\hat{A}_{0}(z)$ and $\hat{A}_{3}(z)$ into a 'Higgs' field $\Phi(z)=\frac{1}{2}\left(\hat{A}_{0}(z)-i \hat{A}_{3}(z)\right)$. The next step is to seek solutions of the dimensionally reduced self-duality (or Hitchin) equations. In the one-instanton sector this is rather straightforward since the corresponding Nahm potential is abelian. What is more tricky, however, is to execute the second Nahm transformation to recover the instanton itself. We shall restrict ourselves to the special case of a zero Higgs field. This means that the corresponding instanton will be radially symmetric: local gauge invariants such as the action density depend on $x_{1}, x_{2}$ and $r=\sqrt{x_{0}^{2}+x_{3}^{2}}$ only. With this restriction the self-duality equations are just

$$
\hat{F}_{y \bar{y}}=\partial_{y} \hat{A}_{\bar{y}}-\partial_{\bar{y}} \hat{A}_{y}=0
$$

Here we have used complex coordinates; $y=z_{1}+i z_{2}, \bar{y}=z_{1}-i z_{2}, \partial_{y}=\frac{1}{2}\left(\partial_{z_{1}}-i \partial_{z_{2}}\right)$, $\partial_{\bar{y}}=\frac{1}{2}\left(\partial_{z_{1}}+i \partial_{z_{2}}\right), \hat{A}_{y}=\frac{1}{2}\left(\hat{A}_{1}(z)-i \hat{A}_{2}(z)\right)$ and $\hat{A}_{\bar{y}}=\frac{1}{2}\left(\hat{A}_{1}(z)+i \hat{A}_{2}(z)\right)$. Consider the ansatz

$$
\hat{A}_{y}=\partial_{y} \phi, \quad \hat{A}_{\bar{y}}=-\partial_{\bar{y}} \phi
$$


which gives $\hat{F}_{y \bar{y}}=-2 \partial_{y} \partial_{\bar{y}} \phi$. Then, in order to satisfy (ब), $\phi$ must be harmonic except at two singularities, since we are aiming for an $S U(2)$ instanton. A suitable $\phi$ satisfies

$$
\left(\partial_{z_{1}}^{2}+\partial_{z_{2}}^{2}\right) \phi(z)=-2 \pi \kappa\left[\delta^{2}(z-\omega)-\delta^{2}(z+\omega)\right]
$$

where $\kappa$ is a constant and $\pm \omega$ are the positions of the two singularities (we have used translational invariance to shift the 'centre of gravity' of the singularities to the origin). The delta functions should be read as periodic (with respect to $z_{1} \rightarrow z_{1}+2 \pi / L_{1}$ and $\left.z_{2} \rightarrow z_{2}+2 \pi / L_{2}\right)$. Physically, the Nahm potential describes two Aharonov-Bohm fluxes of strength $\kappa$ and $-\kappa$ threading the dual torus. They must have equal and opposite strength to ensure a periodic $\hat{A}$. We may assume that $\kappa$ lies between 0 and 1 since it is possible via a (singular) gauge transformation to shift $\kappa$ by an integer amount (under such a transformation the total flux through $\tilde{\mathbb{T}}^{2}$ remains zero).

One can write $\phi$ explicitly in terms of Jacobi theta functions

$$
\phi(z)=\frac{\kappa}{2}\left(\log \frac{\left|\theta\left(\left(y+\omega_{1}+i \omega_{2}\right) \frac{L_{1}}{2 \pi}+\frac{1}{2}+\frac{i L_{1}}{2 L_{2}}, \frac{i L_{1}}{L_{2}}\right)\right|^{2}}{\left|\theta\left(\left(y-\omega_{1}-i \omega_{2}\right) \frac{L_{1}}{2 \pi}+\frac{1}{2}+\frac{i L_{1}}{2 L_{2}}, \frac{i L_{1}}{L_{2}}\right)\right|^{2}}+\frac{i L_{1} L_{2} \omega_{2}}{\pi}(y-\bar{y})-2 \omega_{2} L_{1}\right) .
$$

The theta function is defined as

$$
\theta(w, \tau)=\sum_{n=-\infty}^{\infty} e^{i \pi n^{2} \tau+2 \pi i n w}, \quad \operatorname{Im} \tau>0
$$

and has the periodicity properties $\theta(w+1, \tau)=\theta(w, \tau)$ and $\theta(w+\tau, \tau)=e^{-i \pi \tau-2 \pi i w} \theta(w, \tau)$. In each cell $\theta(w, \tau)$ has a single zero located at the centre of the torus $\left(w=\frac{1}{2}+\frac{1}{2} \tau\right)$. We have chosen the constant term in (7) so that the integral of $\phi$ over the dual torus is zero. This renders $\phi(z)$ an odd function, $\phi(-z)=-\phi(z)$. The Nahm potential derived from (元) was obtained in [5] via the ADHM construction. Here the flux strength $\kappa$ is related to the ADHM 'size', $\lambda$, of an instanton centred at $x_{\mu}=0$,

$$
\kappa=\frac{\pi \lambda^{2}}{L_{1} L_{2}} \text {. }
$$

Although we do not directly use the ADHM formalism in the present paper the relation (9) proves useful in interpreting our results.

The $S U(2)$ instanton we seek, $A_{\mu}(x)$, is the Nahm transform of the abelian potential $\hat{A}(z)$

$$
A_{\mu}^{p q}(x)=\int_{\tilde{T}^{2}} d^{2} z \psi^{p \dagger}(z ; x) \frac{\partial}{\partial x^{\mu}} \psi^{q}(z ; x)
$$


where the $\psi^{p}(z ; x), p=1,2$ are orthonormal zero modes of

$$
-\frac{i}{2} D_{x}^{\dagger}(\hat{A})=\left(\begin{array}{cc}
\frac{1}{2} \bar{x}_{\perp} & \partial_{y}+\partial_{y} \phi-\frac{i}{2} \bar{x}_{\|} \\
\partial_{\bar{y}}-\partial_{\bar{y}} \phi-\frac{i}{2} x_{\|} & \frac{1}{2} x_{\perp}
\end{array}\right) .
$$

In addition to the complex coordinates $y, \bar{y}$ on $\tilde{\mathbb{T}}^{2}$ we have introduced two sets of complex coordinates for $\mathbb{T}^{2} \times \mathbb{R}^{2}$; in the 'parallel' directions $x_{\|}=x_{1}+i x_{2}, \bar{x}_{\|}=x_{1}-i x_{2}$, and in the 'transverse' non-compact directions $x_{\perp}=x_{0}+i x_{3}, \bar{x}_{\perp}=x_{0}-i x_{3}$.

When $x_{\perp}=0$ the Weyl equation decouples and the two zero modes have a simple form 5

$$
\psi^{1}(z ; x)=\left(\begin{array}{c}
0 \\
e^{-\phi(z)} G_{+}(z-\omega)
\end{array}\right), \quad \psi^{2}(z ; x)=\left(\begin{array}{c}
e^{\phi(z)} G_{-}(z+\omega) \\
0
\end{array}\right)
$$

where $G_{ \pm}(z)$ are periodic Green's functions satisfying

$$
\left(-i \partial_{y}-\frac{1}{2} \bar{x}_{\|}\right) G_{+}(z)=\frac{1}{2} \delta^{2}(z), \quad\left(-i \partial_{\bar{y}}-\frac{1}{2} x_{\|}\right) G_{-}(z)=\frac{1}{2} \delta^{2}(z)
$$

$G_{-}(z)$ has a theta function representation

$$
G_{-}(z)=\frac{i L_{1}}{4 \pi^{2}} e^{\frac{1}{2} i x_{\|}(\bar{y}-y)} \frac{\theta^{\prime}\left(\frac{1}{2}+\frac{i L_{1}}{2 L_{2}}, \frac{i L_{1}}{L_{2}}\right) \theta\left(y+\frac{1}{2}+\frac{i L_{1}}{2 L_{2}}-\frac{i}{L_{2}} x_{\|}, \frac{i L_{1}}{L_{2}}\right)}{\theta\left(\frac{1}{2}+\frac{i L_{1}}{2 L_{2}}-\frac{i}{L_{2}} x_{\|}, \tau\right) \theta\left(y+\frac{1}{2}+\frac{i L_{1}}{2 L_{2}}, \frac{i L_{1}}{L_{2}}\right)},
$$

with $\theta^{\prime}(w, \tau)=\partial_{w} \theta(w, \tau)$. The corresponding result for $G_{+}(z)$ can be obtained via $G_{+}(z)=G_{-}^{*}(-z)$. The zero modes have square-integrable singularities at both fluxes:

\begin{tabular}{c|c|c|}
\hline \hline & $z \sim \omega$ & $z \sim-\omega$ \\
\hline$\left|\psi^{1}\right|^{2}$ & $\propto\left|y-\omega_{1}-i \omega_{2}\right|^{2(\kappa-1)}$ & $\propto\left|y+\omega_{1}+i \omega_{2}\right|^{-2 \kappa}$ \\
\hline$\left|\psi^{2}\right|^{2}$ & $\propto\left|y-\omega_{1}-i \omega_{2}\right|^{-2 \kappa}$ & $\propto\left|y+\omega_{1}+i \omega_{2}\right|^{2(\kappa-1)}$ \\
\hline \hline
\end{tabular}

Table 1: Singularities of the $x_{\perp}=0$ zero modes

There are two values of $x_{\|}$(in each copy of $\mathbb{T}^{2}$ ) for which Tab. 1 does not hold. When $x_{\|}=0$ the Green's function's $G_{ \pm}$do not exist. This does not mean there are no zero modes, indeed one can see that

$$
\psi^{1}(z ; x=0)=\left(\begin{array}{c}
0 \\
e^{-\phi(z)}
\end{array}\right), \quad \psi^{2}(z ; x=0)=\left(\begin{array}{c}
e^{\phi(z)} \\
0
\end{array}\right),
$$


are solutions of the $x_{\|}=0$ Weyl equation. $\psi^{1}$ has the expected square-integrable singularity at $z=-\omega$, but for $z=\omega, \psi^{1}(z ; x=0)$ is zero. On the other hand $\psi^{2}(z, x=0)$ diverges at $z=\omega$ but not at $z=-\omega$. The finiteness of $G_{-}(2 \omega)$ was used to derive Tab. 1 . But if it is zero, $\psi^{1}$ will lose its singularity at $z=-\omega$. From the theta function representation of $G_{-}(z)$ one can see that there is exactly one value of $x_{\|}$in $\mathbb{T}^{2}$ where this occurs, $\pi x_{\|}=$ $-i L_{1} L_{2}\left(\omega_{1}+i \omega_{2}\right)$. We wish to investigate whether these 'soft' points are constituent locations.

Notice that the singularity profiles of $\psi^{1}$ and $\psi^{2}$ are exchanged under the replacement $\kappa \rightarrow 1-\kappa$. This suggests that the constituents are exchanged under this mapping. That is, if there are indeed lumps at the two points, then $\kappa \rightarrow 1-\kappa$ swaps the two lumps. The following result formalises this idea

$$
F_{\mu \nu}\left(x_{\|}, x_{\perp}, \kappa\right)=V^{-1}(x) F_{\mu \nu}\left(-x_{\|}+\frac{L_{1} L_{2}}{i \pi}\left(\omega_{1}+i \omega_{2}\right),-x_{\perp}, 1-\kappa\right) V(x),
$$

where $V(x)$ is some $U(2)$ gauge transformation. The proof of Eq. (16) goes as follows: make the change of variables $z \rightarrow-z$ in (10). The zero mode $\psi^{p}(-z ; x)$ satisfies the same Weyl equation as $\psi^{p}(z ; x)$ except that the signs of $\kappa$ and the $x_{\mu}$ are flipped. Under periodic gauge transformations $\hat{A}(z,-\kappa)$ and $\hat{A}(z, 1-\kappa)$ are equivalent up to a constant potential

$$
\hat{A}_{\mu}(z,-\kappa)=U^{-1}(z)\left(\partial_{\mu}+\hat{A}_{\mu}(z, 1-\kappa)+B_{\mu}\right) U(z)
$$

where $\pi B_{1}=-i L_{1} L_{2} \omega_{2}$ and $\pi B_{2}=i L_{1} L_{2} \omega_{1}$. In the Weyl equation $B_{\mu}$ can be absorbed into $x_{1}$ and $x_{2}$. Thus $U^{-1}(z) \psi^{p}\left(z,-x_{\|}-i L_{1} L_{2}\left(\omega_{1}+i \omega_{2}\right) / \pi,-x_{\perp}\right)$ satisfies the same Weyl equation as $\psi^{p}\left(-z ; x_{\|}, x_{\perp}\right)$.

We have established that any lumps centred at the constituent points have a simple exchange property. To actually see the lumps we must compute the field strengths. We can do this explicitly in the $x_{\perp}=0$ slice, since here we have the exact zero modes (12). Before we insert these modes into (10), they must be normalised. This can be done by dividing both modes by $\sqrt{\rho}$, where

$$
\rho=\int_{\tilde{T}^{2}} d^{2} z e^{2 \phi(z)}\left|G_{-}(z+\omega)\right|^{2} .
$$

In fact, one can write two components of the gauge potential solely in terms of this normalisation factor

$$
A_{x_{||}}=-\frac{1}{2} \tau_{3} \partial_{x_{||}} \log \rho, \quad A_{\bar{x}_{||}}=\frac{1}{2} \tau_{3} \partial_{\bar{x}_{||}} \log \rho,
$$


so that

$$
F_{x_{||} \bar{x}_{||}}=\tau_{3} \partial_{x_{||}} \partial_{\bar{x}_{||}} \log \rho
$$

The computation of the other components is more involved since the derivatives of the zero modes with respect to $x_{\perp}$ are required. However, the field strengths can be more easily accessed via Green's function techniques. Here we just quote the results; more details will be given elsewhere

$$
F_{x_{||} \bar{x}_{\perp}}=2 \pi i\left(\tau_{1}+i \tau_{2}\right) \kappa \rho \partial_{x_{||}}^{2} \frac{\nu}{\rho}
$$

where

$$
\nu=\int_{\tilde{T}^{2}} d^{2} z G_{+}(\omega-z) e^{2 \phi(z)} G_{-}(z+\omega) .
$$

The other components are fixed by self-duality, i.e. $F_{x_{\perp} \bar{x}_{\perp}}+F_{x_{||} \bar{x}_{||}}=0$ and $F_{x_{||} x_{\perp}}=0$. Note that $\rho$ is dimensionless, real and periodic (with respect to $x_{1} \rightarrow x_{1}+L_{1}, x_{2} \rightarrow x_{2}+L_{2}$ ), while $\nu$ is dimensionless, complex and periodic up to constant phases. Both $\rho$ and $\nu$ diverge at $x_{\|}=0$, but the field strengths should not. A careful analysis of $\rho$ and $\nu$ in the neighbourhood of $x_{\|}=0$ shows that the field strengths are well defined at this point. Alternatively, one can just note that $\rho$ and $\nu$ are well behaved at the second soft point and invoke (16).

Since we have all field strengths within the $x_{\perp}=0$ slice we can compute the action density

$$
-\frac{1}{2} \operatorname{Tr} F_{\mu \nu} F^{\mu \nu}=16\left(\partial_{x_{||}} \partial_{\bar{x}_{||}} \log \rho\right)^{2}+128 \pi^{2} \kappa^{2} \rho^{2}\left|\partial_{x_{||}}^{2} \frac{\nu}{\rho}\right|^{2} .
$$

We have provided integral representations of $\rho$ and $\nu$ where the integrands are expressible in terms of standard functions. Using these results, we have made numerical plots of the action density for various values of $\kappa$ and $\omega$, and for the periods we have taken $L_{1}=L_{2}=2 \pi$, i.e. a square torus. The most symmetric case, $\kappa=\frac{1}{2}$ and $\omega_{1}=\omega_{2}=\frac{1}{4}$ is plotted in Fig. 11. Two equal sized lumps are observed, one at $x_{\|}=0$, the other at $x_{\|}=(1+i) \pi$. Decreasing $\kappa$ at fixed $\omega$ reduces the size of the $x_{\|}=0$ constituent but increases its contribution to the action. Conversely, the second constituent becomes larger but contributes less to the action density. These two effects tend to (rather quickly) flatten the second peak as $\kappa$ is reduced. Here we provide plots with $\kappa=\frac{7}{16}$ (Fig. 2) and $\kappa=\frac{3}{8}$ (Fig. 3), again with $\omega_{1}=\omega_{2}=\frac{1}{4}$. Already at $\kappa=\frac{3}{8}$ we see that the $x_{\|}=(1+i) \pi$ constituent is more of a plateau than a peak. If $\kappa$ is decreased much further the first peak will dominate completely; this peak 
will be essentially that of a BPST instanton on $R^{4}$ with a scale parameter given by the ADHM formula (9). Indeed, if we take (9) at face value, and use (16), our two constituents are BPST instanton cores, one with scale $\lambda=\sqrt{\kappa L_{1} L_{2} / \pi}$ at $x_{\|}=x_{\perp}=0$, the other with scale $\lambda=\sqrt{(1-\kappa) L_{1} L_{2} / \pi}$ at the second soft point. When $L_{1}=L_{2}$ at least one $\lambda$ is of the order of the size of the torus in line with the strong overlap seen in the plots.

The peaks are best resolved at $\kappa=\frac{1}{2}$, when the constituents are identical. The overlap can be avoided by taking one period, say $L_{2}$, smaller than the other, and then taking a sufficiently large constituent separation in the $x_{1}$ direction. However, then the size of one or both cores will exceed $L_{2}$, tending to suppress or smooth out the dependence on $x_{2}$. In this case the constituents become periodic monopoles. Actually, it is possible to see some hint of this monopole limit even for a square torus; taking $\omega_{1}=\frac{1}{4}, \omega_{2}=0, \kappa=\frac{1}{2}$ with $L_{1}=L_{2}=2 \pi$ the two constituents (at $x_{\|}=0$ and $x_{\|}=i \pi$ ) overlap so strongly that they merge into a monopole worldline, in that the dependence of the field strengths on $x_{2}$ is very weak.

The $\kappa=\frac{1}{2}$ case has another interesting feature. If we choose the constituent locations so that they are separated by half periods the charge one instanton can be 'cut' to yield a charge $\frac{1}{2}$ instanton (see also [7]). This happens when $\left(\omega_{1}, \omega_{2}\right)$ is $\left(\frac{1}{2} \pi / L_{1}, 0\right),\left(0, \frac{1}{2} \pi / L_{2}\right)$ or $\left(\frac{1}{2} \pi / L_{1}, \frac{1}{2} \pi / L_{2}\right)$; the former and the latter are shown in Fig. 4. After cutting we have a twist $Z_{12}=-1$ in the half torus. But to get a half-integer topological charge we also require a twist in the non-compact directions, $Z_{03}=-1$, since non-orthogonal twists are a prerequisite for fractional instanton number. Far away from $x_{\perp}=0$, the potential must be a pure gauge

$$
A_{\mu}(x) \rightarrow V^{-1}\left(x_{1}, x_{2}, \theta\right) \partial_{\mu} V\left(x_{1}, x_{2}, \theta\right) \quad r \rightarrow \infty
$$

where $x_{\perp}=r e^{i \theta}$. The non-compact twist, $Z_{03}=-1$, translates into a double-valued gauge function, $V\left(x_{1}, x_{2}, \theta+2 \pi\right)=-V\left(x_{1}, x_{2}, \theta\right)$. However, the explicit results we have given are restricted to the $r=0$ slice. What can we say about the full $r \neq 0$ potential? It can be argued that as for $r=0$ (as well as $S U(2)$ calorons) two functions, one real the other complex, are sufficient

$$
\begin{aligned}
& A_{x_{||}}=-\frac{\tau_{3}}{2} \partial_{x_{||}} \log \rho-2 \pi i\left(\tau_{1}-i \tau_{2}\right) \kappa \rho \partial_{\bar{x}_{\perp}} \frac{\nu^{*}}{\rho} \\
& A_{x_{\perp}}=-\frac{\tau_{3}}{2} \partial_{x_{\perp}} \log \rho+2 \pi i\left(\tau_{1}-i \tau_{2}\right) \kappa \rho \partial_{\bar{x}_{||}} \frac{\nu^{*}}{\rho} .
\end{aligned}
$$


Here $\rho$ and $\nu$ depend on $r$. The $\rho$ and $\nu$ respectively given in (18) and (22) should be understood as the zeroth order terms of power series in $r^{2}$ for the 'full' $\rho$ and $\nu$. Assuming that $\nu$ falls off exponentially for large $r$, a decay $\rho \sim r^{-1}$, would account for the twist.

Doubly periodic charge $\frac{1}{2}$ instantons have also been found in simulations [8, 9]. Many qualitative features reported are in accord with our findings. In particular, we agree that these objects are single lumps with radial symmetry, and that the action density is never zero in the $x_{\perp}=0$ plane. Another striking observation in [8, 9] is that the action density decays exponentially for large $r$. To make a comparison here, more information about the large $r$ properties of $\rho$ and $\nu$ is required.

\section{Acknowledgements}

We thank F. Bruckmann, M. Engelhardt, O. Jahn, T. Tok and P. van Baal for helpful discussions. C.F. was supported through a European Community Marie Curie Fellowship (contract HPMF-CT-2000-00841).

\section{References}

[1] K. M. Lee and C. h. Lu, Phys. Rev. D 58 (1998) 025011 hep-th/9802108.

[2] T. C. Kraan and P. van Baal, Nucl. Phys. B 533 (1998) 627 hep-th/9805168.

[3] P. J. Braam and P. van Baal, Commun. Math. Phys. 122 (1989) 267.

[4] M. Jardim, Commun. Math. Phys. 216 (2001) 1 math.dg/9909069.

[5] C. Ford, J. M. Pawlowski, T. Tok and A. Wipf, Nucl. Phys. B 596 (2001) 387 hepth/0005221].

[6] H. Reinhardt, O. Schroeder, T. Tok and V. C. Zhukovsky, hep-th/0203012.

[7] A. Gonzalez-Arroyo, Nucl. Phys. B 548 (1999) 626 [hep-th/9811041.

[8] A. Gonzalez-Arroyo and A. Montero, Phys. Lett. B 442 (1998) 273 hep-th/9809037.

[9] A. Montero, Phys. Lett. B 517 (2001) 142 hep-lat/0104008. 


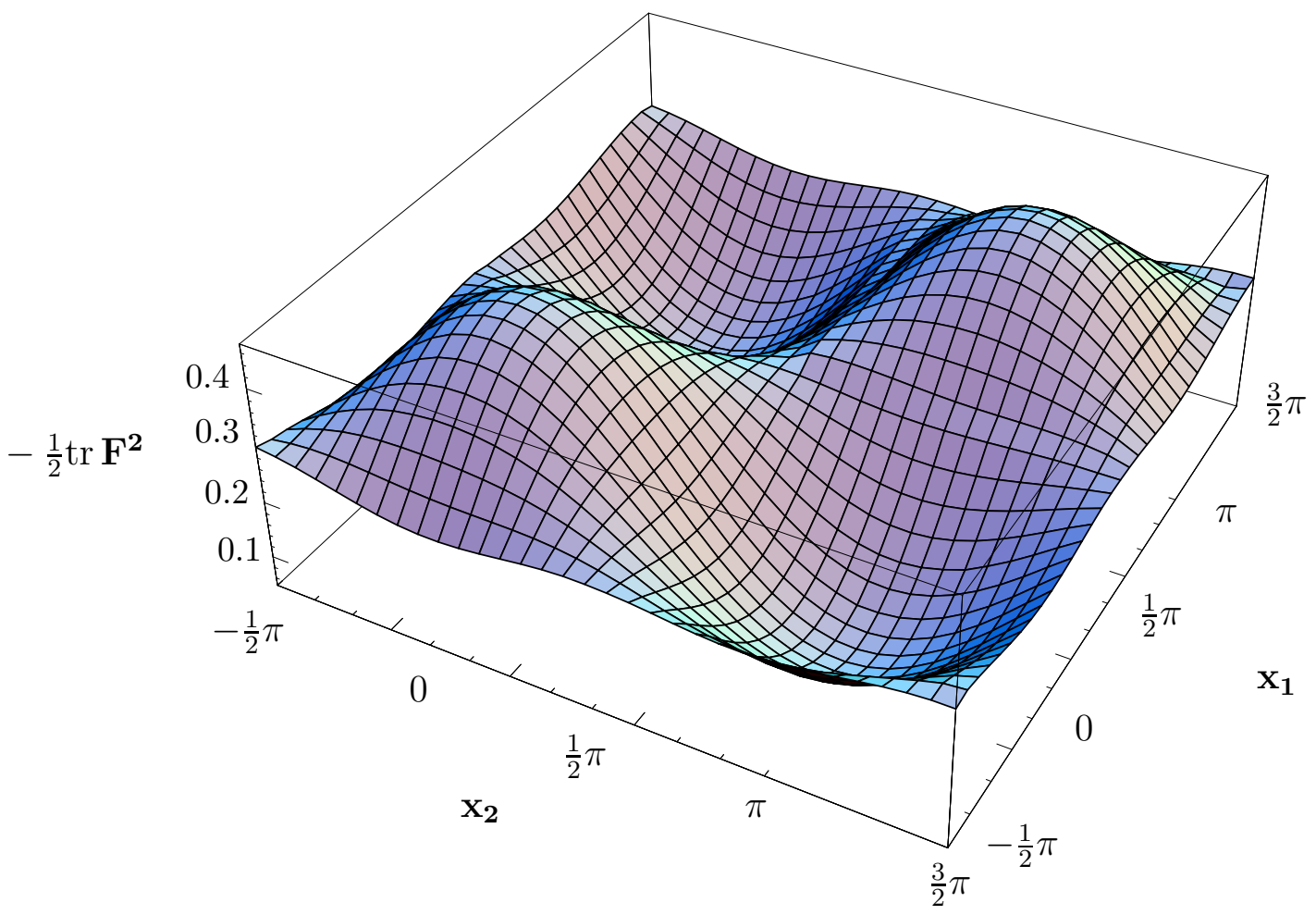

Figure 1: Plot of action density for $x_{\perp}=0$ and $\kappa=\frac{1}{2}$ and $\omega_{1}=\omega_{2}=\frac{1}{4}$.

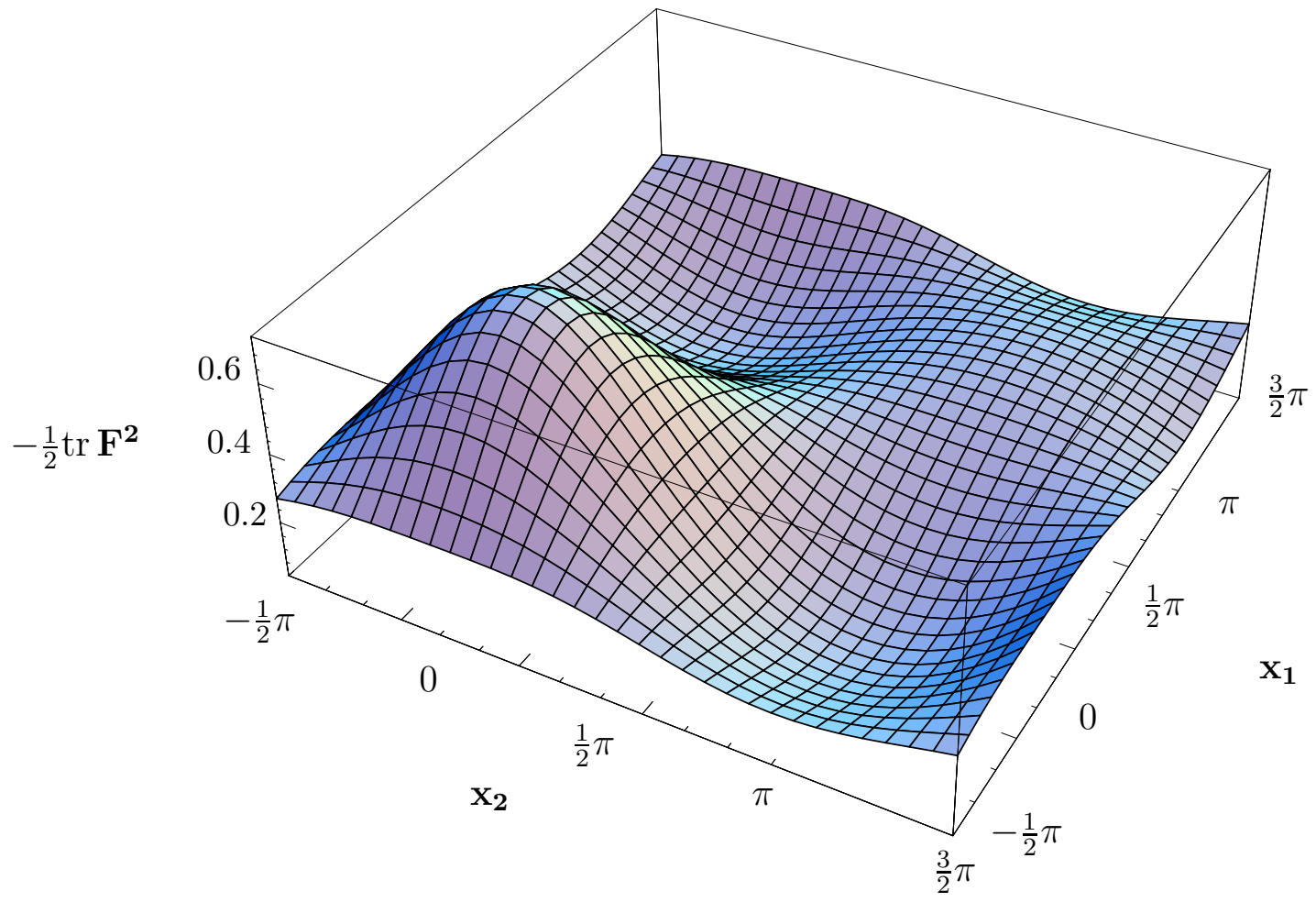

Figure 2: Plot of action density for $x_{\perp}=0$ and $\kappa=\frac{7}{16}$ and $\omega_{1}=\omega_{2}=\frac{1}{4}$. 


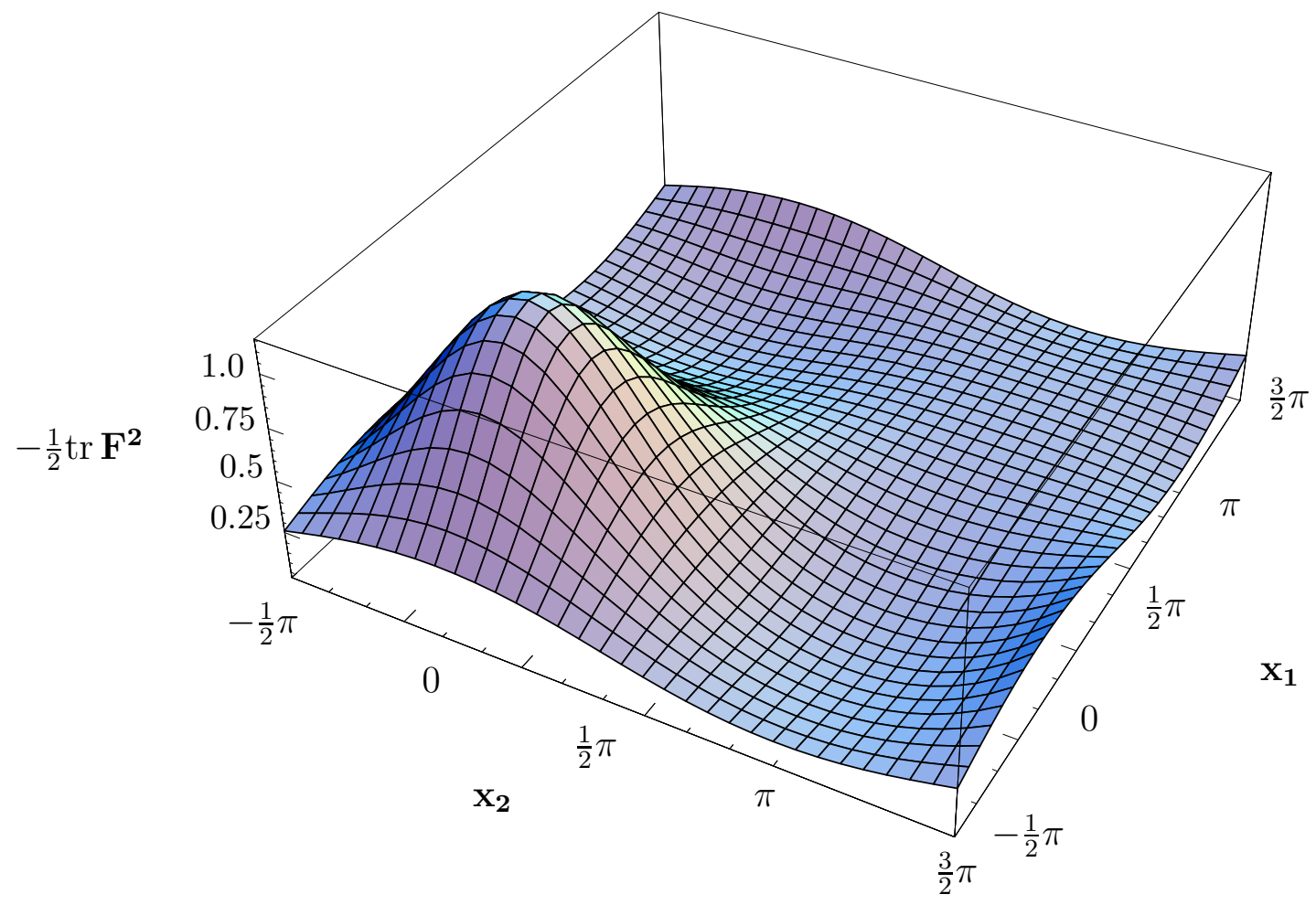

Figure 3: Plot of action density for $x_{\perp}=0$ and $\kappa=\frac{3}{8}, \omega_{1}=\omega_{2}=\frac{1}{4}$.
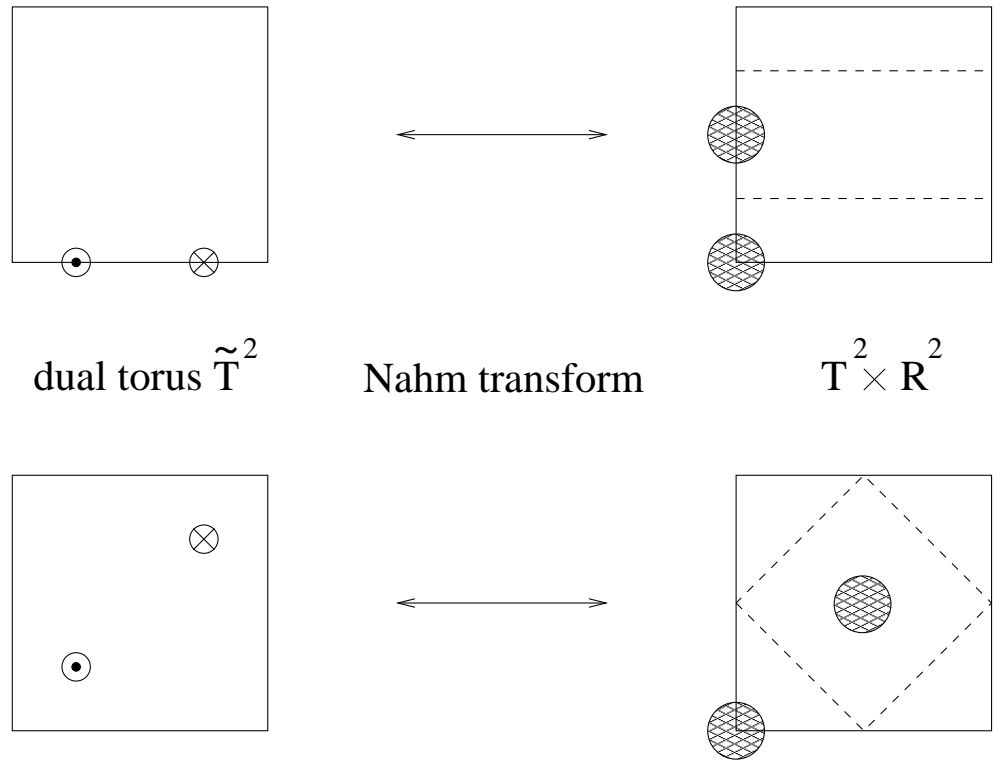

Figure 4: When $\kappa=\frac{1}{2}$, the choices $\omega_{1}=\frac{1}{2} \pi / L_{1}, \omega_{2}=0$ and $\omega_{1}=\frac{1}{2} \pi / L_{1}, \omega_{2}=\frac{1}{2} \pi / L_{2}$ yield constituent separations allowing the torus to be cut (dashed line) to yield charge $\frac{1}{2}$ instantons. 